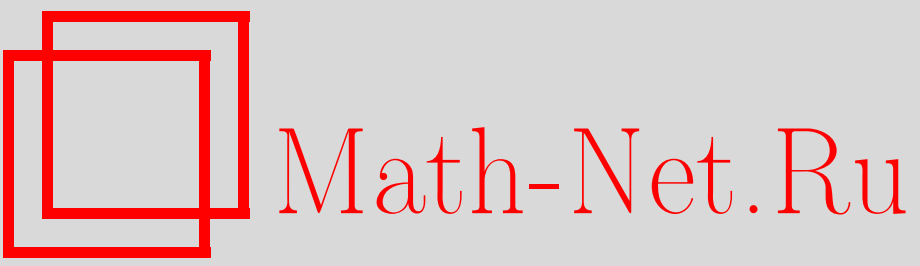

Г. А. Мартынов, Термодинамика и гидродинамика (статистическое обоснование). 3. Уравнения гидродинамики, ТМФ, 2003, том 134, номер 3, 487-500

DOI: https://doi.org/10.4213/tmf166

Использование Общероссийского математического портала Math-Net.Ru подразумевает, что вы прочитали и согласны с пользовательским соглашением http: //www.mathnet.ru/rus/agreement

Параметры загрузки:

IP: 54.174 .149 .18

26 апреля 2023 г., 13:45:33 
ТЕОРЕТИЧЕСКАЯ

И МАТЕМАТИЧЕСКАЯ

ФИЗИКА

Том 134, № 3

март, 2003

(C) 2003 г.

\author{
Г. А. Мартынов*
}

\title{
ТЕРМОДИНАМИКА И ГИДРОДИНАМИКА (СТАТИСТИЧЕСКОЕ ОБОСНОВАНИЕ). 3. УРАВНЕНИЯ ГИДРОДИНАМИКИ
}

Показано, что из иерархии ББГКИ могут быть получены все уравнения гидродинамики. Теория строится путем разложения функций распределения в ряд по малому параметру $\varepsilon=R / L \leqslant 10^{-8}$, где $R \approx 10^{-7}$ см - радиус корреляционной сферы, $L-$ характерный макроскопический размер. Показано, что в нулевом порядке по этому параметру из иерархии ББГКИ следуют состояние локального равновесия и уравнения переноса идеальной жидкости Эйлера; в первом порядке по $\varepsilon$ из иерархии ББГКИ следуют уравнения гидродинамики вязкой жидкости. При этом доказано, что поток внутренней энергии должен включать в себя как поток кинетической энергии, пропорциональный градиенту температуры, так и поток потенциальной энергии, пропорциональный градиенту плотности. Показано, что уравнения гидродинамики справедливы при $t \gg \tau \approx 10^{-12} \mathrm{c}, L \gg R \approx 10^{-7} \mathrm{~cm}$.

Ключевые слова: иерархия ББГКИ, законы сохранения, малый параметр, уравнения гидродинамики.

\section{1. ВВЕДЕНИЕ}

В работах [1], [2] было показано, что в случае полного термодинамического равновесия, при котором все функции распределения перестают зависеть от времени, из иерархии ББГКИ может быть получено распределение Гиббса и как следствие первый закон термодинамики; в данной статье мы продолжим анализ иерархии и покажем, что в случае малых отклонений от равновесия из нее следуют уравнения гидродинамики.

Вывод уравнений гидродинамики из первых принципов (т.е. из иерархии ББГКИ) впервые был дан Боголюбовым в 1948 году [3]. Однако эти уравнения были получены им только в первом приближении по малому параметру. Поэтому они оказались справедливыми в случае идеальной жидкости, в которой эффектами вязкости можно пренебречь. И хотя в конце статьи Боголюбов пишет, что из иерархии ББГКИ в следуюшем приближении по тому же параметру могут быть найдены уравнения гидродинамики вязкой жидкости, доказательство этого утверждения, насколько мне известно,

${ }^{*}$ Институт физической химии РАН, Москва, Россия. E-mail: gamartyn@mtu-net.ru 
в литературе отсутствует. Поэтому отсутствуют и точные формулы для расчета коэффициентов переноса. В отличие от этого в статье Чо и Уленбека 1958 года [4] на основе иерархии ББГКИ были получены точные выражения для уравнений баланса массы, импульса и внутренней энергии, не связанные с какими бы то ни было разложениями по малому параметру. Однако и в этой работе проблема не была решена до конца - из точных, но незамкнутых уравнений баланса не были получены приближенные, но замкнутые уравнения гидродинамики (а также не были получены уравнения диффузии и баланса энтропии). Мне не известны подобные исследования и в настояшее время. Возможной причиной этого является то, что физические предпосылки уравнений переноса выглядят столь очевидными, что, казалось бы, ни в каких дополнительных обоснованиях не нуждаются. На самом деле это не совсем так.

При выводе уравнений гидродинамики, основанном на макроскопическом подходе, физический смысл сделанных приближений не вполне очевиден. Это привело к тому, что в одном из трех уравнений гидродинамики - в уравнении теплопроводности - был учтен член, описывающий перенос кинетической энергии частищ (закон Фурье), но не был включен другой член, ответственный за перенос потенциальной энергии. Поэтому некоторые следствия уравнений гидродинамики оказались в противоречии с данными эксперимента (например, в случае гидродинамической теории распространения звука [5]). Очевидно, что подобные ошибки могут возникать и при рассмотрении других явлений. Кроме того, вывод уравнений гидродинамики из первых принципов необходим для выяснения границ применимости макроскопической теории и для разработки методов расчета коэффициентов переноса по заданным значениям потенциалов взаимодействия частищ (обе эти задачи до сих пор так и не решены). Но главным образом этот вьвод необходим для обоснования самой статистической механики, одной из основных составляющих которой являются уравнения переноса. С помощью этих уравнений определяется одночастичная функция распределения, без которой уравнения иерархии ББГКИ оказываются незамкнутыми [1].

\section{2. ЛОКАЛЬНО-РАВНОВЕСНАЯ ИЕРАРХИЯ ББГКИ}

В случае неравновесных газов и жидкостей в системе возникает естественный малый параметр

$$
\varepsilon=\frac{R}{L} \leqslant 10^{-8}
$$

где $R \approx 10^{-7}$ см - радиус корреляционной сферы и $L$ - характерный макроскопический размер (в случае термодинамически равновесных систем $\varepsilon=0$, поскольку размер макроскопического тела $L$, находяшегося в состоянии равновесия, можно увеличивать до бесконечности, ничего при этом не меняя в данной точке). Но этот параметр явным образом не входит в иерархию ББГКИ. Чтобы ввести его, необходимо сначала несколько преобразовать исходные уравнения. 
Как было показано в работе [1], иерархия ББГКИ может быть записана в виде

$$
\begin{gathered}
\frac{\partial \omega_{1}}{\partial t}+\frac{\vec{p}_{1}}{m} \frac{\partial \omega_{1}}{\partial \vec{r}_{1}}=-\vec{F}_{1} \frac{\partial \omega_{1}}{\partial \vec{p}_{1}}-\frac{\partial \vec{F}_{1}}{\partial \vec{p}_{1}} \\
\frac{\partial \Omega_{(l)}}{\partial t}=-\sum_{i=1}^{l}\left\{\frac{\vec{p}_{i}}{m} \frac{\partial \Omega_{(l)}}{\partial \vec{r}_{i}}+\left(\vec{F}_{(l)}-\vec{F}_{i}\right) \frac{\partial \omega_{i}}{\partial \vec{p}_{i}}+\vec{F}_{(l)} \frac{\partial \Omega_{(l)}}{\partial \vec{p}_{i}}+\frac{\partial\left(\vec{F}_{(l)}-\vec{F}_{i}\right)}{\partial \vec{p}_{i}}\right\} .
\end{gathered}
$$

Здесь термические потенциалы $\omega, \Omega_{(l)}$ связаны с функциями распределения $G_{(l)}$ соотношениями

$$
G_{i}=e^{\omega_{i}}, \quad G_{1, \ldots, l}=e^{\Omega_{1, \ldots, l}} \prod_{i=1}^{l} G_{i}, \quad \Omega_{1} \equiv 0,
$$

а силы $\vec{F}_{(l)} \equiv \vec{F}_{1, \ldots, l}^{(i)}$, действуюшие на частищу $i$, заданы формулами

$$
\vec{F}_{1, \ldots, l}^{(i)}=-\frac{\partial U_{1, \ldots, l}}{\partial \vec{r}_{i}}-\rho_{0} \int_{V} d \vec{r}_{l+1} \int_{-\infty}^{+\infty} \frac{\partial \Phi_{i, l+1}}{\partial \vec{r}_{i}} e^{\omega_{l+1}+\left(\Omega_{1, \ldots, l+1}-\Omega_{1, \ldots, l}\right)} \frac{d \vec{p}_{l+1}}{P^{3}},
$$

где $U_{(l)}=\sum_{i, j=1}^{l} \Phi_{i, j}$ - потенциальная энергия группы, состояшей из $l$ частиц, $\Phi_{i, j}=$ $\Phi_{(2)}\left(r_{i, j}\right)$ - потенциал парного взаимодействия частиц $i$ и $j, \rho_{0}$ - нормировочная плотность, $\vec{r}_{i}, \vec{p}_{i}-$ координата и импульс $i$-й частицы, соответственно.

По определению все термические потеншиалы $\Omega_{(l)}$ являются функциями безразмерного локального времени $t / \tau$ (здесь $\tau \approx 10^{-12} \mathrm{c}$ - характерное время хаотизации), безразмерных импульсов $\vec{p}_{i} / P(P=\sqrt{2 \pi m \theta}-$ нормировочный импульс) и безразмерных координат $\vec{r}_{i} / L$. Последние, конечно, должны отсчитываться от одной и той же точки, жестко связанной с лабораторной (инерциальной) системой координат. Поэтому, казалось бы, все $\vec{r}_{i} \approx L$, где $L$ - характерный макроскопический размер системы. Но вспомним, что благодаря условию ослабления корреляший все термические потеншиалы $\Omega_{(l)}$ обрашаются в нуль при $r_{i j}>R$, где $R$ - радиус корреляционной сферы (см. [1], [2]). Поэтому нас должны интересовать только такие значения $\vec{r}_{i}$, при которых расстояния между отдельными частицами $r_{i j}=\left|\vec{r}_{i}-\vec{r}_{j}\right|$ не превьшают радиуса корреляции. Чтобы учесть этот эффект, заметим, что всегда можно положить $\vec{r}_{j}=\vec{r}_{i}+\left(\vec{r}_{j}-\vec{r}_{i}\right)=\vec{r}_{i}+\vec{r}_{j i}$. Поэтому любую функцию координат $l$ частиц можно представить в виде

$$
f_{(l)}=f_{(l)}\left(\vec{r}_{1}, \ldots, \vec{r}_{i}, \ldots, \vec{r}_{l}\right)=f_{(l)}\left(\vec{r}_{i}+\vec{r}_{1 i}, \ldots, \vec{r}_{i}, \ldots, \vec{r}_{i}+\vec{r}_{l i}\right)=f_{(l)}\left(\vec{q}_{i}, \vec{r}_{1 i}, \ldots, \vec{r}_{l i}\right),
$$

где мы выделили произвольную координату $\vec{r}_{i}$, положив $\vec{q}_{i} \equiv \vec{r}_{i}$. Поэтому

$$
\frac{\partial f_{(l)}}{\partial \vec{r}_{i}}=\frac{\partial f_{(l)}}{\partial \vec{q}_{i}}+\sum_{\substack{j=1 \\ j \neq i}}^{l} \frac{\partial f_{(l)}}{\partial \vec{r}_{j i}} \frac{\partial \vec{r}_{j i}}{\partial \vec{r}_{i}}=\frac{\partial f_{(l)}}{\partial \vec{q}_{i}}-\sum_{\substack{j=1 \\ j \neq i}}^{l} \frac{\partial f_{(l)}}{\partial \vec{r}_{j i}} .
$$

Очевидно, что введенные таким образом модули векторов $q_{i} \approx L$ имеют макроскопический размер, а $r_{i j} \leqslant R$ - микроскопический. 
Если теперь подставить (6) в иерархию ББГКИ (2), получим

$$
\begin{gathered}
\frac{\partial \omega_{1}}{\partial t}+\frac{\vec{p}_{1}}{m} \frac{\partial \omega_{1}}{\partial \vec{q}_{1}}=-\vec{F}_{1} \frac{\partial \omega_{1}}{\partial \vec{p}_{1}}-\frac{\partial \vec{F}_{1}}{\partial \vec{p}_{1}} \\
\frac{\partial \Omega_{(l)}}{\partial t}=-\sum_{i=1}^{l}\left\{\frac{\vec{p}_{i}}{m}\left[\frac{\partial \Omega_{(l)}}{\partial \vec{q}_{i}}-\sum_{\substack{j=1 \\
j \neq i}}^{l} \frac{\partial \Omega_{(l)}}{\vec{r}_{i j}}\right]+\right. \\
\left.+\left(\vec{F}_{(l)}-\vec{F}_{i}\right) \frac{\partial \omega_{i}}{\partial \vec{p}_{i}}+\vec{F}_{(l)} \frac{\partial \Omega_{(l)}}{\partial \vec{p}_{i}}+\frac{\partial\left(\vec{F}_{(l)}-\vec{F}_{i}\right)}{\partial \vec{p}_{i}}\right\} .
\end{gathered}
$$

Переходя затем к безразмерным переменным

$$
t^{*}=\frac{t}{T}, \quad \vec{q}^{*}=\frac{\vec{q}}{L}, \quad \vec{r}_{i j}^{*}=\frac{\vec{r}_{i j}}{R}, \quad \vec{p}^{*}=\frac{\vec{p}}{P},
$$

где $T$ - характерное макроскопическое время, получим

$$
\begin{gathered}
\chi\left(\frac{\partial \omega_{1}}{\partial t}+\frac{\vec{p}_{1}}{m} \frac{\partial \omega_{1}}{\partial \vec{q}_{1}}\right)=-\vec{F}_{1} \frac{\partial \omega_{1}}{\partial \vec{p}_{1}}-\frac{\partial \vec{F}_{1}}{\partial \vec{p}_{1}}, \\
\chi \frac{\partial \Omega_{(l)}}{\partial t}=-\sum_{i=1}^{l}\left\{\frac{\vec{p}_{i}}{m}\left[\varepsilon \frac{\partial \Omega_{(l)}}{\partial \vec{q}_{i}}-\sum_{\substack{j=1 \\
j \neq i}}^{l} \frac{\partial \Omega_{(l)}}{\partial \vec{r}_{i j}}\right]+\right. \\
\left.+\left(\vec{F}_{(l)}-\vec{F}_{i}\right) \frac{\partial \omega_{i}}{\partial \vec{p}_{i}}+\vec{F}_{(l)} \frac{\partial \Omega_{(l)}}{\partial \vec{p}_{i}}+\frac{\partial\left(\vec{F}_{(l)}-\vec{F}_{i}\right)}{\partial \vec{p}_{i}}\right\} .
\end{gathered}
$$

В дальнейшем безразмерный параметр $\chi=\tau / T$ для простоты будем считать равным по величине параметру $\varepsilon=R / L$, т.е. положим $\chi=\varepsilon$.

Наконец, полагая

$$
\omega=\omega^{(0)}+\varepsilon \omega^{(1)}, \quad \Omega_{(l)}=\Omega_{(l)}^{(0)}+\varepsilon \Omega_{(l)}^{(1)}, \quad \vec{F}_{(l)}=\vec{F}_{(l)}^{(0)}+\varepsilon \vec{F}_{(l)}^{(1)}
$$

и приравнивая члены одного порядка по $\varepsilon$, получим в нулевом приближении по этому параметру

$$
\begin{gathered}
\vec{F}_{1}^{(0)} \frac{\partial \omega_{1}^{(0)}}{\partial \vec{p}_{1}}+\frac{\partial \vec{F}_{1}^{(0)}}{\partial \vec{p}_{1}}=0 \\
\sum_{i=1}^{l}\left\{-\frac{\vec{p}_{i}}{m} \sum_{\substack{j=1 \\
j \neq i}}^{l} \frac{\partial \Omega_{(l)}^{(0)}}{\partial \vec{r}_{i j}}+\left(\vec{F}_{(l)}^{(0)}-\vec{F}_{(1)}^{(0)}\right) \frac{\partial \omega_{i}}{\partial \vec{p}_{i}}+\vec{F}_{(l)}^{(0)} \frac{\partial \Omega_{(l)}^{(0)}}{\partial \vec{p}_{i}}+\frac{\partial\left(\vec{F}_{(l)}^{(0)}-\vec{F}_{i}^{(0)}\right)}{\partial \vec{p}_{i}}\right\}=0 .
\end{gathered}
$$


Эти уравнения в точности совпадают с уравнениями $([2]-(9))^{1)}$, полученными при рассмотрении состояния полного термодинамического равновесия. Как было показано в ([2]-(22)), из (11) следует, что

$$
G_{(1)}=\frac{\rho(\vec{q})}{\rho_{0}} e^{-p^{2} /(2 m \theta)},
$$

где $m$ - масса частиц и $\theta$ - температура. Но в данном случае мы имеем дело не с глобальным, а с локальным равновесием, при котором физически бесконечно мальй элемент жидкости $\delta V$ (т.е. корреляционная сфера), находяшийся в состоянии локального равновесия, может двигаться со скоростью $\vec{v}$. Поэтому в $\omega^{(0)}=\ln G_{(1)}$ мы должны заменить $\vec{p}$ на $\vec{p}-m \vec{v}$, после чего решение равновесной иерархии (11) принимает вид

$$
\begin{gathered}
\omega^{(0)}=\ln \frac{\rho(\vec{q}, t)}{\rho^{(0)}}-\frac{(\vec{p}-m \vec{v}(\vec{q}, t))^{2}}{2 m \theta(\vec{q}, t)}, \\
\Omega_{(l)}^{(0)}=\Omega_{(l)}^{(0)}\left(r_{i j} ; \rho(\vec{q}, t), \vec{v}(\vec{q}, t), \theta(\vec{q}, t)\right), \quad \vec{F}_{(1)}^{(0)}=0,
\end{gathered}
$$

где гидродинамические переменные $\rho, \vec{v}, \theta$ являются заданными функциями, значения которых определяются уравнениями гидродинамики, а также начальными и граничными условиями к ним. При этом надо иметь в виду, что гидродинамические переменные выделены среди всех других параметров задачи тем, что они выражаются через интегралы от одночастичной функции распределения $G_{(1)}([1]-(8)-(10))$, тогда как значения остальных параметров определяются интегралами от двухчастичной функции распределения $G_{(2)}$. Для последней граничные условия задаются условиями ослабления корреляций, которые, однако, не накладывают никаких ограничений на $G_{(1)}$. По сушеству, формулы ([1]-(8)-(10)), устанавливающие связь между $G_{(1)}$ и макроскопическим состоянием системы (в данной точке $\vec{q}$ и в данный момент $t$ ), заменяют собой условия ослабления корреляций. При этом, как это следует из самого вида формул $([1]-(8)-(10))$, определяемая ими связь между $G_{(1)}$ и $\rho, \vec{v}, \theta$ является параметрической.

Уравнения первого порядка по $\varepsilon$ будут более подробно рассмотрены ниже. Здесь же мы отметим, что все термические потенциалы зависят от макроскопической координаты $\vec{q}_{i} \equiv \vec{q}$ только через $\rho(\vec{q}, t), \vec{v}(\vec{q}, t), \theta(\vec{q}, t)$. Поэтому производные $\partial(\cdot) / \partial \vec{q}_{i}$ в уравнениях первого порядка по $\varepsilon$ должны иметь вид

$$
\begin{aligned}
\frac{\partial \omega}{\partial q_{\alpha}} & =\frac{\partial \omega}{\partial \rho} \frac{\partial \rho}{\partial q_{\alpha}}+\frac{\partial \omega}{\partial \theta} \frac{\partial \theta}{\partial q_{\alpha}}+\frac{\partial \omega}{\partial v_{\beta}} \widetilde{D}_{\alpha \beta}+\frac{\partial \omega}{\partial v_{\alpha}} \frac{\partial v_{\gamma}}{\partial q_{\gamma}} \\
\frac{\partial \Omega_{(l)}}{\partial q_{\alpha}} & =\frac{\partial \Omega_{(l)}}{\partial \rho} \frac{\partial \rho}{\partial q_{\alpha}}+\frac{\partial \Omega_{(l)}}{\partial \theta} \frac{\partial \theta}{\partial q_{\alpha}}+\frac{\partial \Omega_{(l)}}{\partial v_{\beta}} \widetilde{D}_{\alpha \beta}+\frac{\partial \Omega_{(l)}}{\partial v_{\alpha}} \frac{\partial v_{\gamma}}{\partial q_{\gamma}}
\end{aligned}
$$

где тензор деформации скоростей

$$
\begin{aligned}
& D_{\alpha \beta}=\frac{1}{2}\left(\frac{\partial v_{\alpha}}{\partial q_{\beta}}+\frac{\partial v_{\beta}}{\partial q_{\alpha}}\right)=\widetilde{D}_{\alpha \beta}+\delta_{\alpha \beta} \frac{\partial v_{\alpha}}{\partial q_{\beta}} \\
& \widetilde{D}_{\alpha \beta}=\frac{1}{2}\left(\frac{\partial v_{\alpha}}{\partial q_{\beta}}+\frac{\partial v_{\beta}}{\partial q_{\alpha}}-2 \delta_{\alpha \beta} \frac{\partial v_{\alpha}}{\partial q_{\beta}}\right) .
\end{aligned}
$$

\footnotetext{
1) Здесь [2] указывает номер ссылки в списке литературы, (9) - номер формулы в указанной статье.
} 
В (13) значения производных $\partial \rho / \partial q_{\alpha}, \partial \theta / \partial q_{\alpha}, \widetilde{D}_{\alpha \beta}$ и $\partial v_{\gamma} / \partial q_{\gamma}$, как и самих функций $\rho, \vec{v}, \theta$, определяются уравнениями гидродинамики. Поэтому в иерархии ББГКИ они играют роль независимых констант. Если теперь проинтегрировать соотношения (13) по $\vec{r}_{i j}$, эти константы можно вынести за знак интеграла. В результате члены первого порядка по $\varepsilon$ будут иметь вид, аналогичный (13),

$$
\begin{aligned}
& \omega^{(1)}=\omega_{\alpha}^{(\rho)} \frac{\partial \rho}{\partial q_{\alpha}}+\omega_{\alpha}^{(\theta)} \frac{\partial \theta}{\partial q_{\alpha}}+\omega_{\alpha \beta}^{(D)} \widetilde{D}_{\alpha \beta}+\omega^{(v)} \frac{\partial v_{\gamma}}{\partial q_{\gamma}} \\
& \Omega_{(l)}^{(1)}=\Omega_{\alpha}^{(\rho)} \frac{\partial \rho}{\partial q_{\alpha}}+\Omega_{\alpha}^{(\theta)} \frac{\partial \theta}{\partial q_{\alpha}}+\Omega_{\alpha \beta}^{(D)} \widetilde{D}_{\alpha \beta}+\Omega^{(v)} \frac{\partial v_{\gamma}}{\partial q_{\gamma}}
\end{aligned}
$$

где неизвестные функции $\omega_{\alpha}^{(\rho)}, \omega_{\alpha}^{(\theta)}, \omega_{\alpha \beta}^{(D)}, \omega^{(v)}$ и $\Omega_{\alpha}^{(\rho)}, \Omega_{\alpha}^{(\theta)}, \Omega_{\alpha \beta}^{(D)}, \Omega^{(v)}$ зависят только от микроскопических переменных $\vec{r}_{i j}$ и $\vec{p}_{i}$; зависимость от координаты $\vec{q}_{i} \equiv \vec{q}_{i}$, определяющей положение координационной сферы внутри макроскопического объема системы $V$, задается гидродинамическими переменными $\rho(\vec{q}, t), \vec{v}(\vec{q}, t), \theta(\vec{q}, t)$. Так как в равновесии градиенты гидродинамических переменных обращаются в нуль, то при записи решения в виде (15) автоматически обрашаются в нуль и градиентные члены в пределе $t \rightarrow \infty$ (в этом пределе состояние любой замкнутой изолированной системы стремится к состоянию равновесия). Более строгое обоснование формул (15) будет дано ниже, пока же мы продолжим анализ иерархии ББГКИ и ее следствий.

\section{3. ТОЧНЫЕ УРАВНЕНИЯ БАЛАНСА МАССЫ, ИМПУЛЬСА И ВНУТРЕННЕЙ ЭНЕРГИИ}

Как указывалось во введении, в статье [4] с помошью иерархии ББГКИ были получены точные локальные уравнения баланса массы, импульса и внутренней энергии. Приведем эти выражения, так как они нам понадобятся в дальнейшем.

Интегрирование первого уравнения иерархии ББГКИ (2) по импульсам приводит к локальному закону сохранения числа частиц (т.е. к уравнению непрерывности)

$$
\frac{D \rho}{D t}+\rho \frac{\partial v_{\alpha}}{\partial q_{\alpha}}=0
$$

в котором оператор

$$
\frac{D}{D t}=\frac{\partial}{\partial t}+v_{\alpha} \frac{\partial}{\partial q_{\alpha}} .
$$

Умножая уравнение иерархии ББГКИ для одночастичной функции распределения $G_{(1)}$ на $p_{\alpha} / m$ и интегрируя по $\vec{p}$, получим выражение для локального закона сохранения импульса

$$
m \rho \frac{D v_{\alpha}}{D t}=-\frac{\partial P_{\alpha \beta}}{\partial q_{\beta}},
$$

в котором тензор натяжений $P_{\alpha \beta}$ распадается на две компоненты: кинетическую и потенциальную,

$$
P_{\alpha \beta}=P_{\alpha \beta}^{\text {кин }}+P_{\alpha \beta}^{\text {пот }} .
$$


Первая из них задается соотношением

$$
P_{\alpha \beta}^{\text {кин }}=\rho_{0} \int_{-\infty}^{+\infty} \frac{\tilde{p}_{\alpha} \tilde{p}_{\beta}}{m} G_{(1)} \frac{d \vec{p}}{P^{3}}=\delta_{\alpha \beta} \rho \theta+\rho_{0} \int_{-\infty}^{+\infty}\left[\frac{\tilde{p}_{\alpha} \tilde{p}_{\beta}}{m}-\delta_{\alpha \beta} \frac{\tilde{p}^{2}}{m}\right] G_{(1)} \frac{d \vec{p}}{P^{3}},
$$

а вторая - соотношением

$$
P_{\alpha \beta}^{\text {пот }}=\frac{1}{2} \rho_{0}^{2} \int_{-\infty}^{+\infty} \frac{d \vec{p}_{1} d \vec{p}_{2}}{P^{6}} \int_{0}^{+\infty} \frac{r_{12 \alpha} r_{12 \beta}}{r_{12}} \frac{\partial \Phi_{(2)}\left(r_{12}\right)}{\partial r_{12}} G_{(2)}\left(\vec{q}, r_{12}, \vec{p}_{1}, \vec{p}_{2}, t\right) r_{12}^{2} d r_{12} .
$$

Наконец, определим внутреннюю энергию $е$ одной частицы с помощью формулы

$$
e(\vec{q}, t)=\frac{3}{2} \theta(\vec{q}, t)+e^{(\Phi)}(\vec{q}, t),
$$

в которой первый член в правой части имеет смысл средней кинетической энергии, а второй - средней потенциальной энергии одной частицы; значение последней определяется формулой [4]

$$
\rho(\vec{q}, t) e^{(\Phi)}(\vec{q}, t)=\int_{-\infty}^{+\infty} \frac{d \vec{p}_{1} d \vec{p}_{2}}{P^{6}} \frac{1}{2} \rho_{0}^{2} \int_{V} \Phi_{(2)}\left(r_{12}\right) G_{(2)}\left(\vec{q}, r_{12}, \vec{p}_{1}, \vec{p}_{2}, t\right) d \vec{q}_{2} .
$$

Для определенной таким образом внутренней энергии из первых двух уравнений иерархии ББГКИ следует локальный закон сохранения

$$
\rho \frac{D e}{D t}=-\frac{\partial J_{\alpha}}{\partial q_{\alpha}}-P_{\alpha \beta} D_{\alpha \beta},
$$

где тензор деформации скоростей был определен выше (14) и имеет вид

$$
D_{\alpha \beta}=\frac{1}{2}\left(\frac{\partial v_{\alpha}}{\partial q_{\beta}}+\frac{\partial v_{\beta}}{\partial q_{\alpha}}\right) .
$$

Поток внутренней энергии состоит из трех компонент:

$$
\vec{J}=\vec{J}^{\text {кин }}+\vec{J}^{\text {пот }}+\vec{J}^{\text {вир }} .
$$

Согласно [4] первой компонентой является поток кинетической энергии (т.е. поток температуры)

$$
J_{\alpha}^{\text {кин }}=-\rho_{0} \int_{-\infty}^{+\infty} \frac{\tilde{p}_{\alpha}}{m} \frac{\tilde{p}^{2}}{2 m} G_{(1)} \frac{d \vec{p}}{P^{3}},
$$

вторая компонента - поток потенциальной энергии

$$
J_{\alpha}^{\text {пот }}=\int_{-\infty}^{+\infty} \frac{\tilde{p}_{\alpha}}{m} \frac{d \vec{p}_{1} d \vec{p}_{2}}{P^{6}} \frac{1}{2} \rho_{0}^{2} \int_{0}^{\infty} \Phi_{12} G_{12} 4 \pi r_{12}^{2} d r_{12},
$$

третья компонента - поток вириала

$$
J_{\alpha}^{\text {вир }}=\int_{-\infty}^{+\infty}\left(\frac{\tilde{p}_{1 \beta}}{m}+\frac{\tilde{p}_{2 \beta}}{m}\right) \frac{d \vec{p}_{1} d \vec{p}_{2}}{P^{6}} \frac{1}{4} \rho_{0}^{2} \int_{0}^{\infty} \frac{r_{12 \alpha} r_{12 \beta}}{r_{12}} \frac{\partial \Phi_{12}}{\partial r_{12}} G_{12} 4 \pi r_{12}^{2} d r_{12} .
$$

Заметим, что в механике потоку вириала соответствует поток работы $\vec{v} \int \vec{q}(\partial \Phi / \partial \vec{q}) d \vec{q}$ совершаемой силовым полем частиц. В приведенных выше выражениях $\overrightarrow{\tilde{p}}=\vec{p}-m \vec{v}-$ хаотическая компонента импульса частиц. Подчеркнем, что все эти формулы являются точными, так как они получены из иерархии ББГКИ путем тождественных преобразований. 


\section{4. УРАВНЕНИЯ ГИДРОДИНАМИКИ}

Заметим теперь, что поскольку в иерархии ББГКИ мы разложили все функции распределения в ряд по степеням малого параметра $\varepsilon$, то же самое необходимо сделать и в уравнениях баланса массы, импульса и внутренней энергии. Последние содержат всего три функции, значения которых явным образом зависят от функций распределения, это $P_{\alpha \beta}, e$ и $\vec{J}$. Именно они и должны быть разложены в ряд по $\varepsilon$; гидродинамические переменные $\rho(\vec{q}, t), \vec{v}(\vec{q}, t), \theta(\vec{q}, t)$, значения которых определяются макроскопическими уравнениями переноса, от $\varepsilon$ не зависят.

Обратимся теперь к приведенным выше формулам, определяюшим $P_{\alpha \beta}, e$ и $\vec{J}$. В них под знаком интеграла стоят функции распределения $G_{(2)}=G_{(2)}^{(0)}\left(1+\varepsilon \Omega_{(2)}^{(1)}\right)$, где функция $\Omega_{(2)}^{(1)}$ задана формулой (15). Поскольку градиенты гидродинамических переменных не зависят от разностей координат $r_{i j}$ и от импульсов отдельных частиц $\vec{p}_{i}$, то в формулах, определяющих тензор давлений и потоки макроскопического импульса и энергии, они могут быть вынесены из-под знака интеграла. После этого сами интегралы, играюшие роль множителей при макроскопических градиентах, приобретают смысл коэффициентов переноса, а функции $P_{\alpha \beta}, e$ и $\vec{J}_{\text {принимают вид }}$

$$
A=A^{(0)}+\varepsilon\left[A_{\rho}^{(1)} \frac{\partial \rho}{\partial \vec{q}}+A_{\theta}^{(1)} \frac{\partial \theta}{\partial \vec{q}}+A_{\widetilde{D}}^{(1)} \widetilde{D}+A_{v}^{(1)} \frac{\partial v_{\alpha}}{\partial q_{\alpha}}\right],
$$

где буквой $A$ обозначена любая из трех функций $P_{\alpha \beta}, e, \vec{J}$. Этих соображений достаточно для преобразования точных, но незамкнутых уравнений баланса массы, импульса и внутренней энергии в замкнутую, но приближенную (с точностью до членов порядка $\varepsilon^{2} \approx 10^{-16}$ ) систему уравнений гидродинамики. Для определения самих коэффициентов переноса необходимо научиться определять функции $\Omega_{(2)}^{(1)}$ с помошью уравнений первого порядка по $\varepsilon$, полученных из иерархии ББГКИ. В дальнейшем будем полагать $\tilde{\rho}=m \rho$, причем знак "тильда" будем опускать.

4.1. Уравнение непрерывности. Первый закон сохранения - закон сохранения массы - имеет вид (16). Так как уравнение (16) содержит только гидродинамические переменные $\rho$ и $\vec{v}$, значения которых в рядне разлагаются, то его видне должен меняться и после разложения остальных уравнений баланса в ряд по $\varepsilon$.

4.2. Уравнение Навье-Стокса. В отличие от закона сохранения массы (16) закон сохранения импульса (18) содержит тензор натяжений $P_{\alpha \beta}$, который можно представить в виде $P_{\alpha \beta}=P_{\alpha \beta}^{(0)}+\varepsilon P_{\alpha \beta}^{(1)}$, где $P_{\alpha \beta}^{(0)}=\delta_{\alpha \beta} P$ и $P=P(\rho, \theta)$ - обычное равновесное давление в жидкости. В частном случае $A=P_{\alpha \beta}$ можно написать

$$
P_{\alpha \beta}=\delta_{\alpha \beta} P+\left(\mu \frac{\partial \rho}{\partial q_{\alpha}}+\nu \frac{\partial \theta}{\partial q_{\alpha}}\right)-\eta \widetilde{D}_{\alpha \beta}-\xi \delta_{\alpha \beta} \frac{\partial v_{\gamma}}{\partial q_{\gamma}} .
$$

В этом выражении константы $\mu, \nu, \eta$ и $\xi$, характеризуюшие свойства вешества, должны быть скалярами, поскольку жидкость предполагается изотропной (проблема получения уравнений переноса для анизотропных сред, например жидких кристаллов, требует особого рассмотрения). По определению градиенты плотности и температуры являются векторами, тогда как $P_{\alpha \beta}$ - тензор. Для того чтобы тензорная размерность всех 
членов (31) была одинаковой, необходимо предположить, что $\mu=\nu=0$ (что, конечно, должно непосредственно следовать из формул, определяющих $\left.P_{\alpha \beta}\right)$. В результате окончательно получаем

$$
P_{\alpha \beta}=\delta_{\alpha \beta} P-\eta \widetilde{D}_{\alpha \beta}-\xi \delta_{\alpha \beta} \frac{\partial v_{\gamma}}{\partial q_{\gamma}}
$$

(знак минус перед двумя последними членами поставлен для того, чтобы коэффициент сдвиговой вязкости $\eta$ и коэффициент объемной вязкости $\xi$ были положительными). Подстановка (32) в уравнение баланса импульса (18) приводит к уравнению Навье-Стокса (см. [6], формула (15.7))

$$
\rho \frac{D v_{\alpha}}{D t}=-P_{\rho} \frac{\partial \rho}{\partial q_{\alpha}}-P_{\theta} \frac{\partial \theta}{\partial q_{\alpha}}+\eta \Delta v_{\alpha}+\left(\xi+\frac{1}{3} \eta\right) \frac{\partial}{\partial q_{\alpha}} \frac{\partial v_{\beta}}{\partial q_{\beta}},
$$

где производные $P_{\rho}=(\partial P / \partial \rho)_{\theta}, P_{\theta}=(\partial P / \partial \theta)_{\rho}$ и коэффициенты вязкости $\eta, \xi$ в обшем случае являются функциями $\rho$ и $\theta$. В (33) мы также заменили полную производную $\partial P / \partial q_{\alpha}$ на сумму $P_{\rho}\left(\partial \rho / \partial q_{\alpha}\right)+P_{\theta}\left(\partial \theta / \partial q_{\alpha}\right)$, для того чтобы выделить явным образом градиенты гидродинамических переменных $\partial \rho / \partial \vec{q}, \partial \theta / \partial \vec{q}$ и отделить их от функций $P_{\rho}(\rho, \theta), P_{\theta}(\rho, \theta)$, зависяших не от градиентов, а от самих $\rho$ и $\theta$.

4.3. Уравнение энергопроводности. Уравнение баланса внутренней энергии (24) содержит три "разлагаемые" функции: внутреннюю энергию $e$, поток внутренней энергии $\vec{J}$ и тензор натяжений $P_{\alpha \beta}$. Преобразование $(24)$ начнем с рассмотрения произведения $P_{\alpha \beta} D_{\alpha \beta}$. Так как $\delta_{\alpha \beta} \widetilde{D}_{\alpha \beta}=0$, то

$$
P_{\alpha \beta} D_{\alpha \beta}=\left[\delta_{\alpha \beta} P-\eta \widetilde{D}_{\alpha \beta}-\delta_{\alpha \beta} \xi \frac{\partial v_{\alpha}}{\partial q_{\beta}}\right]\left(\widetilde{D}_{\alpha \beta}+\frac{\partial v_{\gamma}}{\partial q_{\gamma}}\right)=P \frac{\partial v_{\gamma}}{\partial q_{\gamma}}-\eta \widetilde{D}_{\alpha \beta}^{2}-\xi\left(\frac{\partial v_{\gamma}}{\partial q_{\gamma}}\right)^{2} .
$$

Из уравнения непрерывности (16) следует, что $P\left(\partial v_{\gamma} / \partial q_{\gamma}\right)=-(P / \rho)(D \rho / D t)$. Подставляя это выражение в (34), запишем (24) в виде

$$
\rho \frac{D e}{D t}-\frac{P}{\rho} \frac{D \rho}{D t}=-\frac{\partial J_{\alpha}}{\partial q_{\alpha}}+\eta \widetilde{D}_{\alpha \beta}^{2}+\xi\left(\frac{\partial v_{\gamma}}{\partial q_{\gamma}}\right)^{2} .
$$

Внутреннюю энергию также необходимо записать в виде $e=e^{(0)}+\varepsilon e^{(1)}$. Как мы видели, все члены первого порядка по $\varepsilon$ пропорциональны или градиентам плотности и температуры, или тензору скоростей. А так как энергия является скаляром, то все поправки первого порядка к ней должны равняться нулю, из-за того что они имеют другую тензорную размерность. Поэтому в локально-равновесных системах внутренняя энергия $e=e^{(0)}(\rho, \theta)$ по-прежнему должна определяться формулами обычной равновесной термодинамики. Согласно этим формулам производная $(\partial e / \partial \theta)_{\rho}=c_{V}$, где $c_{V}-$ теплоемкость при постоянном объеме, и $\rho^{2}(\partial e / \partial \rho)_{\theta}=P-\theta(\partial P / \partial \theta)_{\rho}$ (см. [7]). Отсюда следует, что

$$
\rho \frac{D e}{D t}=\rho\left(\frac{\partial e^{(0)}}{\partial \rho}\right)_{\theta} \frac{D \rho}{D t}+\rho\left(\frac{\partial e^{(0)}}{\partial \theta}\right)_{\rho} \frac{D \theta}{D t}=\frac{1}{\rho}\left(P-\theta\left(\frac{\partial P}{\partial \theta}\right)_{\rho}\right) \frac{D \rho}{D t}+\rho c_{V} \frac{D \theta}{D t} .
$$


Подставляя (36) в (35), будем иметь

$$
\rho c_{V} \frac{D \theta}{D t}-\frac{\theta P_{\theta}}{\rho} \frac{D \rho}{D t}=-\frac{\partial J_{\alpha}}{\partial q_{\alpha}}+\eta D_{\alpha \beta}^{2}+\xi\left(\frac{\partial v_{\alpha}}{\partial q_{\alpha}}\right)^{2} .
$$

Наконец, запишем поток внутренней энергии в виде

$$
\vec{J}=\vec{J}^{(0)}+\varepsilon \vec{J}^{(1)}=\vec{J}^{(0)}-\lambda \frac{\partial \theta}{\partial q_{\alpha}}+\chi \frac{\partial \rho}{\partial q_{\alpha}}+\mu \widetilde{D}_{\alpha \beta}+\nu \frac{\partial v_{\alpha}}{\partial q_{\alpha}}
$$

и учтем, что в состоянии равновесия $\vec{J}^{(0)}=0$, из-за того что функции распределения $G_{(l)}^{(0)}=G_{(l)}^{(0)}\left(p^{2}\right)$ являются четными функциями импульсов. Кроме того, из соображений тензорной размерности следует, что $\mu=\nu=0$. В результате получаем выражение для потока внутренней энергии ${ }^{2}$

$$
\vec{J}=-\lambda \frac{\partial \theta}{\partial \vec{q}}+\chi \frac{\partial \rho}{\partial \vec{q}}
$$

Эта формула представляет собой обобшение закона Фурье $\vec{J}=-\lambda(\partial \theta / \partial \vec{q})$, учитывающего только перенос кинетической энергии. Согласно (22) внутренняя энергия, помимо кинетической составляющей $3 \theta / 2$, имеет также потенциальную составляюшую $e^{(\Phi)}$, зависяшую не только от $\theta$, но и от $\rho(23)$. Чтобы определить ее вклад в поток внутренней энергии, вспомним, что плотность вешества тесно связана с его потенциальной энергией, поскольку от $\rho$ зависит среднее расстояние между частицами $\bar{r}_{12} \sim \rho^{1 / 3}$ и, следовательно, среднее значение потенциальной энергии $\overline{e^{(\Phi)}(q)} \approx e^{(\Phi)}(\bar{q})$. Поэтому второй член в (39) представляет собой поток потенциальной энергии. Учитывая это, будем называть коэффициент $\chi$ коэффициентом потенциалопроводности. Однако это название не совсем точно. Действительно, вспомним, что согласно (26)-(29) полный поток внутренней энергии распадается на три компоненты: поток кинетической энергии, поток потенциальной энергии и поток вириала, причем каждая из этих трех составляюших зависит от $\rho$ и $\theta$. Подобная зависимость появляется потому, что в нулевом приближении по $\varepsilon$ двухчастичная функция распределения $G_{(2)}$ оказывается функцией этих параметров. Очевидно, что вириал также дает определенный вклад как в $\lambda$, так и в $\chi$.

В формуле (39) перед градиентом температуры был поставлен знак "минус", потому что кинетическая энергия всегда перетекает от более высокой температуры к более низкой. Следовательно, ее поток направлен против градиента температуры. В то же время потенциальная энергия системы растет с увеличением плотности. Поэтому ее поток направлен по градиенту плотности, что и учитывается знаком "плюс" перед вторым членом. При таком выборе знаков коэффициент теплопроводности $\lambda$ и коэффициент потенциалопроводности $\chi$ становятся положительными константами (что,

\footnotetext{
${ }^{2)}$ Впервые на возможность появления дополнительного члена в выражении для потока тепла было указано в известной монографии Чепмена и Каулинга [8], в которой этот член был получен для газов на основе анализа уравнения Больцмана. Впоследствии этот результат был подтвержден в [9], [10]. В [11] дополнительный член в уравнение для потока тепла был введен на основе более общих соображений путем анализа иерархии ББГКИ.
} 
конечно, должно следовать из формул, определяющих эти коэффициенты). Но, к сожалению, в настоящее время аппарат вычисления коэффищиентов переноса на основе иерархии ББГКИ еще не разработан. Поэтому сейчас приходится ограничиться выражением $\chi=3 \eta P_{\rho} /(2 \rho m)$, полученным в работах [9]-[11] для газов на основе уравнения Больцмана, из которого следует, что $\chi$ действительно является положительной величиной.

Подставляя (39) в (37), окончательно получаем

$$
\rho c_{V} \frac{D \theta}{D t}-\frac{\theta P_{\theta}}{\rho} \frac{D \rho}{D t}=\lambda \Delta \theta-\chi \Delta \rho+\eta \widetilde{D}_{\alpha \beta}^{2} .
$$

Это уравнение обычно называется уравнением теплопроводности, хотя точнее было бы называть его уравнением энергопроводности, так как такого понятия как “тепло” в статистической механике просто не существует.

Заметим также, что если в уравнении непрерывности (16), в уравнении Навье-Стокса (33) и в уравнении энергопроводности (40) опустить члены, пропорциональные $\varepsilon$, мы получим систему уравнений Эйлера, описывающую эволюцию идеальной жидкости,

$$
\begin{gathered}
\frac{D \rho}{D t}=-\rho \frac{\partial v_{\alpha}}{\partial q_{\alpha}} \\
m \rho \frac{D v_{\alpha}}{D t}=-\delta_{\alpha \beta} P_{\rho} \frac{\partial \rho}{\partial q_{\beta}}-\delta_{\alpha \beta} P_{\theta} \frac{\partial \theta}{\partial q_{\beta}} \\
\frac{D \theta}{D t}=\frac{\theta P_{\theta}}{\rho c_{V}} \frac{\partial v_{\alpha}}{\partial q_{\alpha}} .
\end{gathered}
$$

Три уравнения гидродинамики (которые необходимо решать одновременно, поскольку невозможно представить себе систему, в которой нарушался бы хотя бы один из трех законов сохранения) определяют эволюцию во времени и в пространстве трех гидродинамических переменных $\rho, \vec{v}$ и $\theta$. Тем самым они определяют эволюцию вообще всех термодинамических параметров системы $\mathcal{A}(\rho, \vec{v}, \theta)$. Действительно, поскольку теория процессов переноса строится путем разложения всех функций распределения в ряд по параметру $\varepsilon$, то соответствуюшим образом должны быть разложены и все термодинамические параметры $\mathcal{A}=\mathcal{A}^{(0)}+\varepsilon \mathcal{A}^{(1)}$, где $\mathcal{A}^{(0)}$ - локально-равновесное значение $\mathcal{A}$ Но поскольку $\varepsilon \approx 10^{-8}$, то членом с $\mathcal{A}^{(1)}$ практически всегда можно пренебречь. Поэтому в неравновесных системах с точностью до членов порядка $\varepsilon$ все термодинамические параметры вешества $\mathcal{A}$ определяются теми же формулами, что и в равновесной термодинамике и, в частности, все они являются функциями плотности и температуры, $\mathcal{A}=\mathcal{A}^{(0)}[\rho(\vec{q}, t), \theta(\vec{q}, t)]$

\section{5. УРАВНЕНИЯ ИЕРАРХИИ ББГКИ В ПЕРВОМ ПРИБЛИЖЕНИИ ПО $\varepsilon$}

Теперь остается еще доказать справедливость формул (15), с помощью которых были получены уравнения гидродинамики. Для этого необходимо записать уравнения, определяющие $\omega^{(1)}, \Omega_{(l)}^{(1)}$. Здесь мы ограничимся рассмотрением первого уравнения иерархии, так как остальные уравнения преобразуются аналогично. 
Как мы видели, первое уравнение иерархии ББГКИ имеет вид

$$
\varepsilon\left(\frac{\partial \omega_{1}}{\partial t}+\frac{\vec{p}_{1}}{m} \frac{\partial \omega_{1}}{\partial \vec{q}_{1}}\right)=-\vec{F}_{1} \frac{\partial \omega_{1}}{\partial \vec{p}_{1}}-\frac{\partial \vec{F}_{1}}{\partial \vec{p}_{1}}
$$

Разлагая входящие в него функции в ряд по $\varepsilon$ и собирая вместе члены первого порядка, получим

$$
\frac{\partial \omega_{1}^{(0)}}{\partial t}+\frac{\vec{p}_{1}}{m} \frac{\partial \omega_{1}^{(0)}}{\partial \vec{q}_{1}}=-\vec{F}_{1}^{(1)} \frac{\partial \omega_{1}^{(0)}}{\partial \vec{p}_{1}}-\vec{F}_{1}^{(0)} \frac{\partial \omega_{1}^{(1)}}{\partial \vec{p}_{1}}-\frac{\partial \vec{F}_{1}^{(1)}}{\partial \vec{p}_{1}}
$$

Учтем теперь, что $\vec{F}_{1}^{(0)}=0$ и $\omega^{(0)}$ задано формулой (12). Поэтому уравнение (44) можно записать в виде

$$
\frac{\partial \omega_{1}^{(0)}}{\partial t}+\frac{\vec{p}_{1}}{m} \frac{\partial \omega_{1}^{(0)}}{\partial \vec{q}_{1}}=-\frac{\overrightarrow{\tilde{p}}_{1}}{m \theta} \vec{F}_{1}^{(1)}-\frac{\partial \vec{F}_{1}^{(1)}}{\partial \vec{p}_{1}}
$$

где импульс $\overrightarrow{\tilde{p}}=\vec{p}-m \vec{v}$. Это уравнение в левой части содержит производные $\omega^{(0)}$ по $\vec{q}, t$. Чтобы вычислить их, преобразуем левую часть (46) следуюшим образом:

$$
\frac{\partial \omega^{(0)}}{\partial t}+\frac{p_{\alpha}}{m} \frac{\partial \omega^{(0)}}{\partial q_{\alpha}}=\frac{D \omega^{(0)}}{D t}+\frac{\tilde{p}_{\alpha}}{m} \frac{\partial \omega^{(0)}}{\partial q_{\alpha}}
$$

где оператор $D(\cdot) / D t$ задан формулой (17). При вычислении производных по времени $D(\cdot) / D t$ и $\partial \omega^{(0)} / \partial q_{\alpha}$ воспользуемся уравнениями гидродинамики идеальной жидкости (41)-(43), следующими из уравнений баланса массы, импульса и энергии в нулевом приближении по $\varepsilon$; учет членов более высокого порядка был бы эквивалентен учету в исходном уравнении (44) членов порядка $\varepsilon^{2} \approx 10^{-16}$, что лежит за границами применимости теории. Обрашение к макроскопическим уравнениям переноса в данном случае вполне обоснованно, так как эволюция гидродинамических переменных $\rho, \vec{v}, \theta$, а значит, и $\omega^{(0)}$ описывается уравнениями гидродинамики, которые сами являются следствием иерархии ББГКИ.

Проведя вычисления, получим

$$
\begin{aligned}
\frac{\omega^{(0)}}{\partial t}+ & \frac{p_{\alpha}}{m} \frac{\partial \omega^{(0)}}{\partial q_{\alpha}}=\frac{\tilde{p}_{\alpha}}{m}\left(1-\frac{P_{\rho}}{\theta}\right) \frac{1}{\rho} \frac{\partial \rho}{\partial q_{\alpha}}+ \\
& +\frac{\tilde{p}_{\alpha}}{m}\left(\frac{\tilde{p}^{2}}{2 m \theta}-\frac{P_{\theta}}{\rho}\right) \frac{1}{\theta} \frac{\partial \theta}{\partial q_{\alpha}}+\frac{\tilde{p}_{\alpha} \tilde{p}_{\beta}}{m \theta} \widetilde{D}_{\alpha \beta}-\left[1+\left(\frac{2}{3}+\frac{P_{\theta}}{\rho c_{V}}\right) \frac{\tilde{p}^{2}}{2 m \theta}\right] \frac{\partial v_{\alpha}}{\partial q_{\alpha}}= \\
= & \mathrm{a}_{\alpha}(p) \frac{\partial \rho}{\partial q_{\alpha}}+\mathrm{b}_{\alpha}(p) \frac{\partial \theta}{\partial q_{\alpha}}+\mathrm{c}_{\alpha \beta}(p) \widetilde{D}_{\alpha \beta}+\mathrm{d}(p) \frac{\partial v_{\alpha}}{\partial q_{\alpha}}
\end{aligned}
$$

Таким образом, в первом порядке по $\varepsilon$ первое уравнение иерархии ББГКИ записьвается в виде

$$
\mathrm{a}_{\alpha} \frac{\partial \rho}{\partial q_{\alpha}}+\mathrm{b}_{\alpha} \frac{\partial \theta}{\partial q_{\alpha}}+\mathrm{c}_{\alpha \beta} \widetilde{D}_{\alpha \beta}+\mathrm{d} \frac{\partial v_{\gamma}}{\partial q_{\gamma}}=-\frac{\tilde{p}_{\alpha}}{m \theta} F_{\alpha}^{(1)}-\frac{\partial F_{\alpha}^{(1)}}{\partial p_{\alpha}} .
$$

Заметим теперь, что градиенты гидродинамических переменных являются независимыми (друг от друга) параметрами задачи. Поэтому без ограничения обшности можно 
положить их все равными нулю, кроме, скажем, градиента плотности. В этом случае уравнение (49) принимает вид

$$
\frac{\tilde{p}_{\alpha}}{m}\left(1-\frac{P_{\rho}}{\theta}\right) \frac{1}{\rho} \frac{\partial \rho}{\partial q_{\alpha}}=-\frac{\tilde{p}_{\alpha}}{m \theta} \rho^{(0)} \int \frac{\partial \Phi_{12}}{\partial \vec{q}_{1}} \exp \left(\omega_{2}^{(0)}+\Omega_{12}^{(0)}\right)\left(\omega_{2 \alpha}^{(\rho)}+\Omega_{12 \alpha}^{(\rho)}\right) \frac{d \vec{r}_{12} d \vec{p}_{2}}{P^{3}}
$$

где $\omega_{\alpha}^{(\rho)}, \Omega_{\alpha}^{(\rho)}$ - функции, стоящие в (15) множителями при градиенте плотности. Аналогичные уравнения могут быть получены и для других компонент общего решения (т.е. $\omega_{\alpha}^{(\theta)}, \Omega_{\alpha}^{(\theta)}$ и т.д.). Все они отличаются друг от друга только выражениями, стояшими в левой части (50).

\section{6. ЗАКЛЮЧЕНИЕ}

Главный результат данной работы - вывод необратимых уравнений гидродинамики из обратимой иерархии ББГКИ. Это оказалось возможньм только потому, что для преобразования исходных обратимых уравнений иерархии мы воспользовались разложением по параметру $\varepsilon=R / L$. Условие $\varepsilon \ll 1$ всегда выполняется в макроскопических телах, размер которых $L$ много больше радиуса корреляционной сферы $R$. Мы видели, что в нулевом приближении по $\varepsilon$ внутри корреляционной сферы реализуется состояние локального равновесия, при котором система "забывает" начальные условия, т.е. те траектории, по которым частицы пришли к состоянию равновесия. Эта “забывчивость" и преврашает обратимые уравнения иерархии ББГКИ в необратимые уравнения гидродинамики.

Состояние локального равновесия устанавливается в корреляционной сфере не мгновенно, а за времена $\tau \approx 10^{-12}$ с, где $\tau$-время, которое Боголюбов назвал временем хаотизации, имея в виду, что за это время внутри корреляционной сферы устанавливается распределение Максвелла по скоростям. Я бы назвал его временем установления локального равновесия, так как только при $t>\tau$ система становится локально-равновесной, "забывает" свои начальные условия и из обратимой преврашается в необратимую.

Фактически вся теория процессов переноса строится в пределе $t / \tau \rightarrow \infty$, в котором термические потенциалы $\Omega_{(l)}$, описьваюшие распределение плотности внутри корреляционной сферы, перестают зависеть от "местного" времени - они заметно меняются только вместе с изменением гидродинамических переменных $\rho(\vec{q}, t), \vec{v}(\vec{q}, t), \theta(\vec{q}, t)$ на макроскопических временах $t \approx T$ ( $T$ - время релаксации макроскопической системы к состоянию термодинамического равновесия). Эти соображения определяют область применимости гидродинамической теории,

$$
t \gg \tau \approx 10^{-12} \mathrm{c}, \quad L \gg R \approx 10^{-7} \mathrm{~cm} .
$$

Заметим, что авторы иерархии ББГКИ предполагали, что сушествуют два характерных времени: $\tau$ и $\tau^{*}=\sigma / \rho v$, где $\sigma$ - диаметр частиц, $\rho=N \sigma^{3} / V$ - безразмерная плотность и $v$ - тепловая скорость движения частиц, приблизительно равная скорости звука. При этом считалось, что в интервале $\tau^{*}<t<\tau$ состояние системы должно описываться кинетическим уравнением, а при $\tau<t-$ уравнениями гидродинамики. Из приведенных же выше соображений следует, что сушествует всего одно характерное время 
$\tau \approx 10^{-12}$ с и что при $t>\tau$ сразу наступает гидродинамический этап эволюции системы; кинетический этап вообще отсутствует. Соответственно отпадает необходимость и в кинетических уравнениях, описываюших эволюцию системы на кинетическом этапе. Однако это не означает, что кинетические уравнения как таковые вообще не нужны - они необходимы, но лишш для определения вида зависимости одночастичной функции распределения $G_{(1)}$ от импульсов $\vec{p}$ (установление вида зависимости $G_{(1)}(\vec{p})$ необходимо для расчета коэффициентов переноса, входяших в уравнения гидродинамики в качестве материальных констант). При этом надо помнить, что только одно кинетическое уравнение является точным - это сама иерархия ББГКИ; все остальные уравнения (включая и уравнение Больцмана) являются приближенными.

Еще одним важным результатом данной статьи является доказательство того, что уравнение энергопроводности (или, что то же, теплопроводности) должно содержать дополнительный член, описывающий процесс переноса потенциальной энергии частиц.

\section{Список литературы}

[1] Г. А. Мартынов. ТМФ. 2002. Т. 133. № 1. С. 121.

[2] Г. А. Мартынов. ТМФ. 2003. Т. 134. № 2. С. 325.

[3] Н. Н. Боголюбов. Уравнения гидродинамики в статистической механике. В сб.: Избранные труды. Т. 2. Киев: Наукова думка, 1970. С. 258.

[4] С. Чо, Джс. Уленбек. Кинетическая теория явлений в плотных газах. Дополнение к монографии Дж. Уленбека, Дж. Форда "Лекции по статистической механике". М.: Мир, 1965. C. 189.

[5] Г.А. Мартынов. ТМФ. 2002. Т. 129. С. 140.

[6] Л.Д. Ландау, Е. М. Лифииц. Механика сплошных сред. М.: Гостехиздат, 1954.

[7] Л. Д. Ландау, Е. М. Лифшич. Статистическая физика. Ч. 1. М.: Наука, 1976.

[8] С. Чепмен, Т. Каулинг. Математическая теория неоднородных газов. М.: ИЛ, 1960.

[9] Ю. Л. Климонтович. Статистическая теория открытых систем. Т. 1. М.: Янус, 1995.

[10] В. М. ЖҚданов, В. И. Ролдугин. УФН. 1998. Т. 168. С. 408.

[11] Г. А. Мартынов. УФН. 1996. Т. 166. С. 1105. после доработки 19.II.2002 г. 